\title{
INDEX TO VOLUME 14
}

Arzhantsev, Ivan V., and Hausen, Jürgen On the multiplication map of a

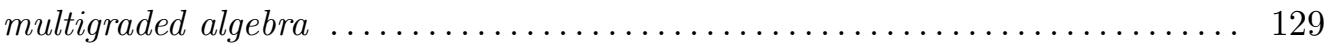

Avila, Artur, and Bochi, Jairo Generic expanding maps without absolutely

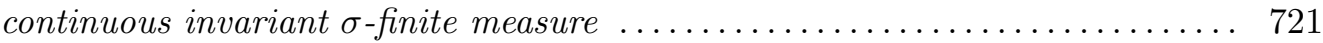

Barbieri-Viale, Luca Formal Hodge theory .................... 385

Behrend, Kai, and Bryan, Jim Super-rigid Donaldson-Thomas invariants . . 559

Bernard, Patrick Smooth critical sub-solutions of the Hamilton-Jacobi equation 503

Bloom, Thomas, and Shiffman, Bernard Zeros of random polynomials on

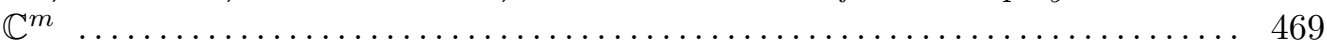

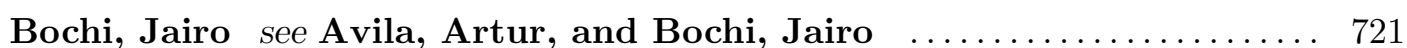

Bodin, Arnaud, and Pichon, Anne Meromorphic functions, bifurcation sets

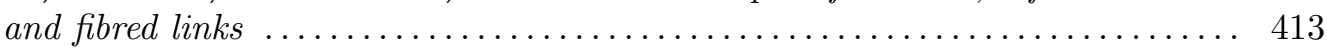

Bownik, Marcin Connectivity and density in the set of framelets ........ 285

Bridson, Martin R., and Howie, James Subgroups of direct products of two

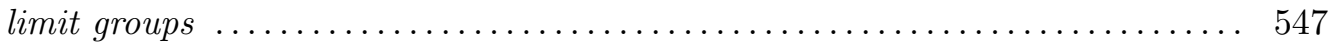

Bringmann, Kathrin, Conley, Charles H., and Richter, Olav K. Maass-

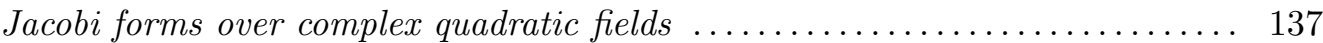

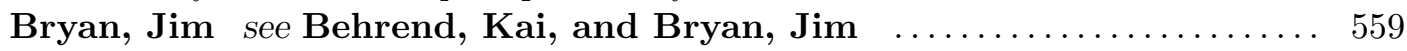

Burq, Nicolas, and Hitrik, Michael Energy decay for damped wave equations

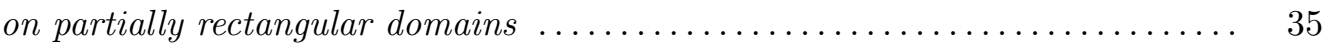

Chaput, Pierre-Emmanuel, and Fu, Baohua On stratified Mukai flops . . . 1055

Ciliberto, Ciro, Lopes, Margarida Mendes, and Pardini, Rita Surfaces with $K^{2}<3 \chi$ and finite fundamental group . . . . . . . . . . . . . . . . 1069

Clark, Pete L. Abelian points on algebraic varieties ................ 731

Conley, Charles H. see Bringmann, Kathrin, Conley, Charles H., and

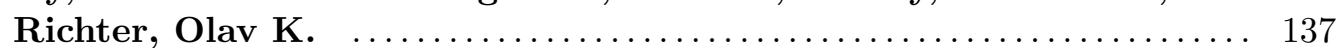

Cozzi, Elaine An initial value problem for two-dimensional ideal incompressible fluids with continuous vorticity ........................ 573

Derenthal, Ulrich On a constant arising in Manin's conjecture for Del Pezzo

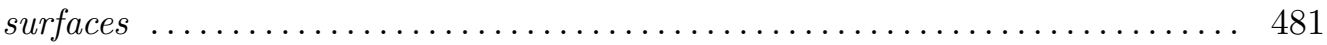

Dewaghe, Laurent Périodicité des polynômes de division sur une courbe ellip-

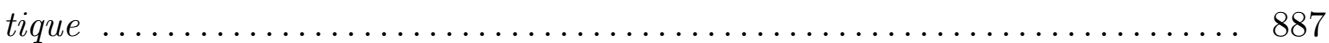

Diaconescu, D. E., Donagi, R., and Pantev, T. Intermediate Jacobians and ADE Hitchin Systems ................................ 745

Dolgushev, Vasiliy Formality theorem for Hochschild (co)chains of the algebra

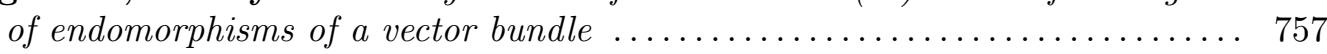

Donagi, R. see Diaconescu, D. E., Donagi, R., and Pantev, T. ...... 745

Ellenberg, Jordan S., and Logan, Adam Asymptotics of coinvariants of Iwasawa modules under non-normal subgroups .................. 769 
Etingof, Pavel, and Eu, Ching-Hwa Koszulity and the Hilbert series of preprojective algebras ......................................

Etnyre, John B. Contact structures on 3-manifolds are deformations of foliations 775

Eu, Ching-Hwa see Etingof, Pavel, and Eu, Ching-Hwa ........... 589

Feigin, Boris, and Shoikhet, Boris On $[A, A] /[A,[A, A]]$ and on a $W_{n}$-action on the consecutive commutators of free associative algebras ............ 781

Fels, Gregor Locally homogeneous finitely nondegenerate CR-manifolds ...... 893

Fine, Joel Fibrations with constant scalar curvature Kähler metrics and the CMline bundle ............................................ 239

Fisher, Tom A new approach to minimising binary quartics and ternary cubics 597

Forstnerič, Franc, and Slapar, Marko Deformations of Stein structures and extensions of holomorphic mappings ......................... 343

Freitas, Pedro, and Krejčiřík, David Unbounded planar domains whose second nodal line does not touch the boundary ........................ 107

Fu, Baohua see Chaput, Pierre-Emmanuel, and Fu, Baohua ......... 1055

Furusho, Hidekazu, and Jafari, Amir Algebraic Cycles and Motivic Generic Iterated Integrals ....................................... 923

Galatolo, Stefano Dimension and hitting time in rapidly mixing systems . . . 797

Galindo, César, and Natale, Sonia Simple Hopf algebras and deformations of

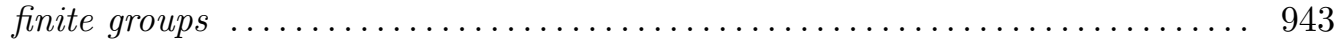

Garoufalidis, Stavros, and Nakamura, Hiroaki Corrigendum : Some IHXtype relations on trivalent graphs and symplectic representation theory ..... 689

Geschke, Stefan, and Kojman, Menachem Metric Baumgartner theorems

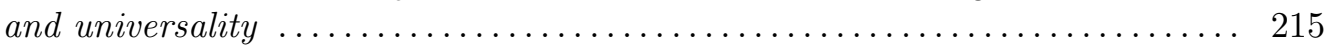

Globevnik, Josip Degree and holomorphic extensions ................... 615

González, Ana, Lupercio, Ernesto, Segovia, Carlos, Uribe, Bernardo, and Xicoténcatl, Miguel Chen-Ruan Cohomology of cotangent orbifolds and Chas-Sullivan String Topology ............................... 491

González-Avilés, Cristian D., and Tan, Ki-Seng A generalization of the Cassels-Tate dual exact sequence ............................. 295

Granja, A. The valuative tree of a two-dimensional regular local ring ........ 19

Gruher, Kate A duality between string topology and the fusion product in equi-

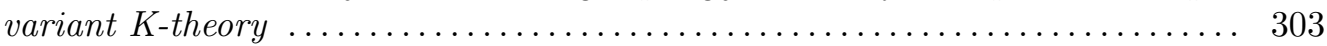

Guillemin, V., Paul, T., and Uribe, A. "Bottom of the well" semi-classical

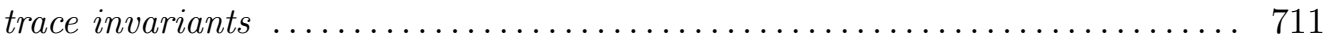

Guillemin, Victor, and Uribe, Alejandro Some inverse spectral results for semi-classical Schrödinger operators .......................... 623

Hang, Fengbo On the integral systems related to Hardy-Littlewood-Sobolev in-

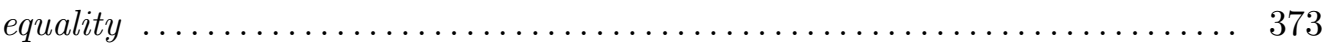

Hartmann, Julia, and Shepler, Anne V. Reflection Groups and Differential Forms ............................................... 955

Hausen, Jürgen see Arzhantsev, Ivan V., and Hausen, Jürgen _...... 129

Hitrik, Michael see Burq, Nicolas, and Hitrik, Michael .............. 35

Howie, James see Bridson, Martin R., and Howie, James ........... 547

Huggins, Bonnie Fields of moduli of hyperelliptic curves .................. 249 
Ilyashenko, Yu. Variation of argument and Bernstein index for holomorphic

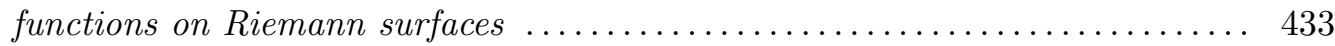

Jafari, Amir see Furusho, Hidekazu, and Jafari, Amir . . . . . . ... 923

Kan, Su-Jen Some complete invariant metrics in Grauert tubes ........... 633

Kapovich, Ilya, Rivin, Igor, Schupp, Paul, and Shpilrain, Vladimir Densities in free groups and $\mathbb{Z}^{k}$, visible points and test elements ....... 263

Karshon, Yael, and Kessler, Liat Circle and torus actions on equal symplectic

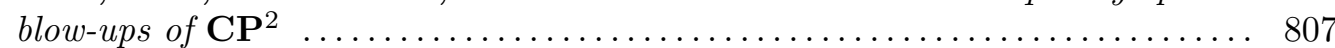

Katz, Sheldon, Li, Wei-Ping, and Qin, Zhenbo On certain moduli spaces of ideal sheaves and Donaldson-Thomas invariants ................. 403

Kessler, Liat see Karshon, Yael, and Kessler, Liat $\ldots \ldots \ldots \ldots \ldots \ldots . . .607$

Kimura, Kenichiro Nori's construction and the second Abel-Jacobi map . . . . 973

Koch, Herbert, Smith, Hart F., and Tataru, Daniel Sharp Lq bounds on spectral clusters for Holder metrics . ....................... 77

Kojman, Menachem see Geschke, Stefan, and Kojman, Menachem . . 215

Krejčiř́k, David see Freitas, Pedro, and Krejčiřík, David . ....... 107

Kumar, N. Mohan, Rao, A. P., and Ravindra, G. V. Generators for vector bundles on generic hypersurfaces .......................... 649

Lackenby, Marc Adding high powered relations to large groups . ......... 983

Laghi, Norberto, and Lyall, Neil Strongly singular integral operators associated to different quasi-norms on the Heisenberg group .................. 825

Li, Wei-Ping see Katz, Sheldon, Li, Wei-Ping, and Qin, Zhenbo ..... 403

Liu, Kefeng, and $\mathbf{X u}$, Hao New properties of the intersection numbers on moduli

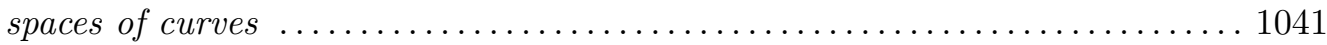

Liu, Kefeng, and Ma, Xiaonan A remark on 'Some numerical results in complex differential geometry' ............................. 165

Logan, Adam see Ellenberg, Jordan S., and Logan, Adam . ....... 769

Lopes, Margarida Mendes see Ciliberto, Ciro, Lopes, Margarida Mendes, and Pardini, Rita ................................... 1069

Lupercio, Ernesto see González, Ana, Lupercio, Ernesto, Segovia, Carlos, Uribe, Bernardo, and Xicoténcatl, Miguel ............... 491

Lyall, Neil see Laghi, Norberto, and Lyall, Neil f............. 825

Ma, Xiaonan see Liu, Kefeng, and Ma, Xiaonan $\ldots \ldots \ldots \ldots \ldots \ldots \ldots$

Macrì, Emanuele Stability conditions on curves ................. 657

Maire, Christian Some new evidence for the Fontaine-Mazur conjecture . . . . 673

Manolescu, Ciprian An unoriented skein exact triangle for knot Floer homology 839

Margalit, Dan, and Spallone, Steven A homological recipe for pseudo-Anosovs 853

Martin, Joaquim, and Milman, Mario Sharp Gagliardo-Nirenberg inequalities

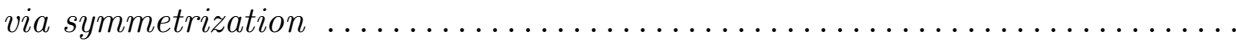

Miao, Pengzi A Note on existence and non-existence of horizons in some asymp-

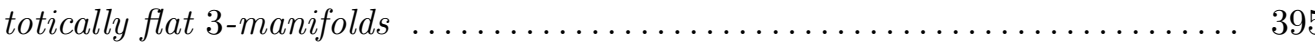

Milman, Mario see Martin, Joaquim, and Milman, Mario ......... 49

Mok, Chung Pang Sato-Tate distribution for abelian varieties with real multiplication over function fields $\ldots \ldots \ldots \ldots \ldots \ldots \ldots \ldots \ldots \ldots \ldots \ldots \ldots \ldots \ldots$ 
Mombelli, Juan Martín, and Natale, Sonia Tensor categories and vacant double groupoids.

Monod, Nicolas Vanishing up to the rank in bounded cohomology ........... 681

Munteanu, Ovidiu Two results on the weighted Poincaré inequality on complete Kähler manifolds .......................................... 995

Nakamura, Hiroaki see Garoufalidis, Stavros, and Nakamura, Hiroaki 689

Natale, Sonia see Mombelli, Juan Martín, and Natale, Sonia ......... 1

Natale, Sonia see Galindo, César, and Natale, Sonia ................ 943

Oberlin, Richard Bounds for Kakeya-type maximal operators associated with

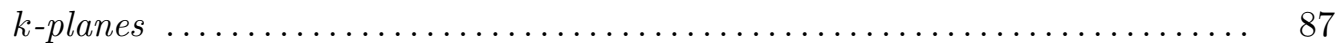

Oblomkov, Alexei Deformed Harish-Chandra homomorphism for the cyclic

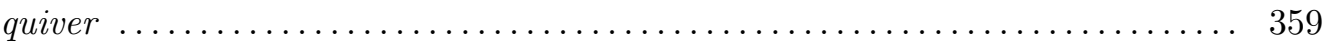

Olsson, Martin A boundedness theorem for Hom-stacks .................. 1009

Ornea, Liviu, and Verbitsky, Misha Embeddings of compact Sasakian manifolds .............................................. 703

Panchishkin, Alexei, and Vankov, Kirill Explicit Shimura's conjecture for $S p_{3}$ on a computer ..................................... 173

Pantev, T. see Diaconescu, D. E., Donagi, R., and Pantev, T. ....... 745

Pardini, Rita see Ciliberto, Ciro, Lopes, Margarida Mendes, and Pardini,

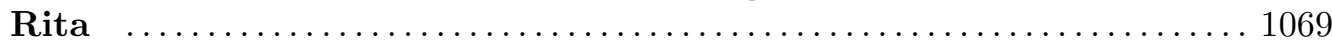

Paul, T. see Guillemin, V., Paul, T., and Uribe, A. .............. 711

Pichon, Anne see Bodin, Arnaud, and Pichon, Anne ............. 413

Plans, Bernat Generic Galois extensions for $\mathrm{SL}_{2}\left(\mathbb{F}_{5}\right)$ over $\mathbb{Q} \ldots \ldots \ldots \ldots . . . . .443$

Poonen, Bjorn Gonality of modular curves in characteristic $p$............. 691

Popescu, Cristian D. Stark's question on special values of $L$-Functions ..... 531

Popescu, Ionel Talagrand Inequality for the Semicircular Law and Energy of the Eigenvalues of Beta Ensembles ................................ 1023

Qin, Zhenbo see Katz, Sheldon, Li, Wei-Ping, and Qin, Zhenbo ...... 403

Rafi, Kasra Thick-thin decomposition for quadratic differentials ............ 333

Ramakrishnan, Dinakar, and Shahidi, Freydoon Siegel modular forms of genus 2 attached to elliptic curves .............................. 315

Rao, A. P. see Kumar, N. Mohan, Rao, A. P., and Ravindra, G. V. . . 649

Ravindra, G. V. see Kumar, N. Mohan, Rao, A. P., and Ravindra, G.

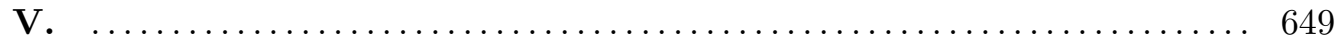

Richter, Olav K. see Bringmann, Kathrin, Conley, Charles H., and Richter, Olav K. ............................... 137

Rivin, Igor see Kapovich, Ilya, Rivin, Igor, Schupp, Paul, and Shpilrain,

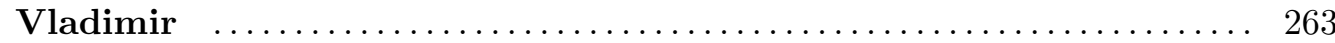

Ross, J. Seshadri constants on symmetric products of curves $\ldots . \ldots \ldots \ldots \ldots .63$

Rouse, Jeremy Atkin-Serre type conjectures for automorphic representations on

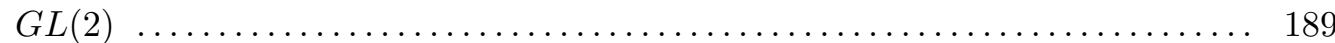

Schupp, Paul see Kapovich, Ilya, Rivin, Igor, Schupp, Paul, and Shpilrain, Vladimir ..................................... 263

Segovia, Carlos see González, Ana, Lupercio, Ernesto, Segovia, Carlos, Uribe, Bernardo, and Xicoténcatl, Miguel _................. 491

Shahidi, Freydoon see Ramakrishnan, Dinakar, and Shahidi, Freydoon 315 
Shen, Zhongwei A relationship between the Dirichlet and regularity problems for elliptic equations ....................................... 205

Shepler, Anne V. see Hartmann, Julia, and Shepler, Anne V. ...... 955

Shiffman, Bernard see Bloom, Thomas, and Shiffman, Bernard . . . . 469

Shoikhet, Boris see Feigin, Boris, and Shoikhet, Boris $\ldots . \ldots \ldots \ldots \ldots \ldots 781$

Shpilrain, Vladimir see Kapovich, Ilya, Rivin, Igor, Schupp, Paul, and Shpilrain, Vladimir f............................... 263

Slapar, Marko see Forstnerič, Franc, and Slapar, Marko _........... 343

Smith, Hart F. see Koch, Herbert, Smith, Hart F., and Tataru, Daniel $\quad 77$

Spallone, Steven see Margalit, Dan, and Spallone, Steven .......... 853

Tan, Ki-Seng see González-Avilés, Cristian D., and Tan, Ki-Seng . . . . 295

Tataru, Daniel see Koch, Herbert, Smith, Hart F., and Tataru, Daniel 77

Tosatti, Valentino, and Weinkove, Ben The Calabi flow with small initial

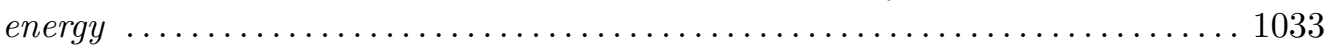

Tzermias, Pavlos Almost rational torsion points and the cuspidal torsion packet on Fermat quotient curves ..................................... 99

Ulmer, Douglas Jacobi sums, Fermat Jacobians, and ranks of abelian varieties over towers of function fields .............................. 453

Urban, Roman, and Zienkiewicz, Jacek Weak type $(1,1)$ estimates for a class

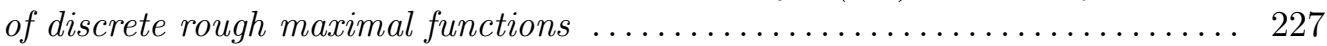

Uribe, A. see Guillemin, V., Paul, T., and Uribe, A. ............ 711

Uribe, Alejandro see Guillemin, Victor, and Uribe, Alejandro ....... 623

Uribe, Bernardo see González, Ana, Lupercio, Ernesto, Segovia, Carlos, Uribe, Bernardo, and Xicoténcatl, Miguel ................. 491

Vankov, Kirill see Panchishkin, Alexei, and Vankov, Kirill ......... 173

Verbitsky, Misha see Ornea, Liviu, and Verbitsky, Misha ........... 703

Wei, Si-Ming, and $\mathbf{X u}$, Hong-Wei Scalar curvature of minimal hypersurfaces

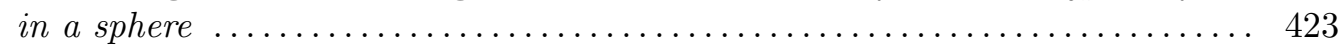

Weinkove, Ben see Tosatti, Valentino, and Weinkove, Ben _........ 1033

Wu, Hao On Legendrian surgeries ............................... 513

Xavier, Frederico An index formula for Loewner vector fields ............. 865

Xia, Changyu The Caffarelli-Kohn-Nirenberg Inequalities on Complete Manifolds 875

Xicoténcatl, Miguel see González, Ana, Lupercio, Ernesto, Segovia, Carlos, Uribe, Bernardo, and Xicoténcatl, Miguel _............. 491

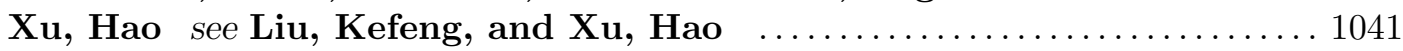

Xu, Hong-Wei see Wei, Si-Ming, and Xu, Hong-Wei $\ldots \ldots \ldots \ldots \ldots 423$

Zarhin, Yuri G. Abelian varieties without homotheties ................... 157

Zienkiewicz, Jacek see Urban, Roman, and Zienkiewicz, Jacek ...... 227 